\title{
Asphyxie Perinatale Au Service De Neonatologie De L'hopital De La Paix De Ziguinchor (Senegal)
}

\section{Thiam L. \\ Dramé A. \\ Coly IZ.}

Université Assane Seck de Ziguinchor, Hôpital de la paix de Ziguinchor Diouf FN.

Université Assane Seck de Ziguinchor, Centre hospitalier régional de Ziguinchor

Sylla A.

Université Cheikh Anta Diop de Dakar, Hôpital Aristite Le Dantec de Dakar

Ndiaye $O$.

Université Cheikh Anta Diop de Dakar, Hôpital d'enfants Albert Royer de

Dakar

doi: 10.19044/esj.2017.v13n21p217 URL:http://dx.doi.org/10.19044/esj.2017.v13n21p217

\begin{abstract}
Introduction: Perinatal asphyxia (PA) is one of the reasons for the transfer of the newborn to neonatal resuscitation services. The objective of this work was to study the epidemiological, obstetrical, clinical and evolutionary aspects of PA at the Ziguinchor Peace Hospital. Materials and methods: This was a retrospective study of cases of hospitalized HAs during the period 1 December 2015 to 31 May 2017. The diagnosis of PA was based on an anomaly of the rhythm Fetal heart rate and / or amniotic fluid abnormality; A score of Apgar $<7$ at the 5th minute and / or the absence of an immediate cry at birth. We collected epidemiological, obstetric, clinical and evolutionary data. Results: We collected 153 cases of PA (90 boys and 63 girls) among 742 newborns, an incidence of $20.6 \%$ of neonatal hospitalizations. The mean age of mothers was 17.2 years; $82.0 \%$ of mothers were between 18 and 35 years of age and half $(50.3 \%)$ had four or more NPCs. Anemia (37.0\%), maternal infection (36.4\%), hypertension (30.9\%), premature rupture of membranes (RPM) $(28.6 \%)$, eclampsia and Preeclampsia $(9.9 \%)$ were the main obstetric complications. The delivery was performed by Caesarean section in $52.3 \%$ of cases and by low procedure in $47.7 \%$ of cases. The term of birth was between 37 and 41 SA in $53.3 \%$ of the cases. The amniotic fluid (LA) was tinted in $55.7 \%$ of the cases and
\end{abstract}


meconium in $12.3 \%$. At the 5th minute, $42.6 \%$ of the infants had an Apgar score $<7$. One hundred percent of the children $(n=153)$ had been aspirated at birth and 24.8\% $(\mathrm{n}=38)$ had been ventilated To the mask. One hundred and twenty-three patients $(80.3 \%)$ had anoxo-ischemic encephalopathy, of which $95(77.2 \%)$ were in stage $1 ; 27(22.0 \%)$ were in stage 2 and $1(0.8 \%)$ was in stage 3 of the Sarnat classification. The main neurological signs were the anomalies of archaic reflexes (64.2\%); Tone disorders (57.7\%); Disorders of consciousness (22.8\%); Convulsions $(21.9 \%)$. The case fatality rate was $10.4 \%$ (16 deaths). Deaths were significantly associated with low birth, MMP, mask ventilation, respiratory distress, and convulsion. Conclusion: PA accounts for $20.6 \%$ of neonatal hospitalizations at the Ziguinchor Peace Hospital. Its lethality is $10.4 \%$. It is associated with high morbidity.

Keywords: Perinatal asphyxia; Sarnat classification; Morbidity and mortality

\section{Resume}

Introduction : L'asphyxie périnatale (AP) constitue l'un des motifs de transfert du nouveau-né vers les services de réanimation néonatale. L'objectif de ce travail était d'étudier les aspects épidémiologiques, obstétricaux, cliniques et évolutifs de l'AP à l'hôpital de la paix de Ziguinchor. Matériels et méthodes : Il s'agissait d'une étude rétrospective portant sur les cas d'AP hospitalisés durant la période allant du $1^{\text {er }}$ décembre 2015 au 31 mai 2017. Le diagnostic d'AP était fait sur la base d'une anomalie du rythme cardiaque fœetal et/ou d'une anomalie du liquide amniotique ; d'un score d'Apgar < 7 à la 5éme minute et/ou de l'absence d'un cri immédiat à la naissance. Nous avons recueilli les données épidémiologiques, obstétricales, cliniques et évolutives. Résultats : Nous avons colligé 153 cas d'AP (90 garçons et 63 filles) parmi 742 nouveau-nés, soit une incidence de $20,6 \%$ des hospitalisations en néonatologie. L'âge moyen des mères était de 17,2 ans ; 82,0\% des mères avaient un âge compris entre 18 et 35 ans et la moitié d'entre elles (50,3\%) avaient fait $4 \mathrm{CPN}$ ou plus. L'anémie (37,0\%), l'infection maternelle (36,4\%), l'HTA (30,9\%), la rupture prématurée des membranes (RPM) $(28,6 \%)$, l'éclampsie et la prééclampsie $(9,9 \%)$ étaient les principales complications obstétricales. L'accouchement était fait par césarienne dans 52,3\% des cas et par voie basse dans $47,7 \%$ des cas. Le terme de naissance se situait entre 37 et $41 \mathrm{SA}$ dans 53,3\% des cas. Le liquide amniotique (LA) était teinté dans 55,7\% des cas et méconial dans 12,3\%. A la 5éme minute $42,6 \%$ des nouveau-nés avaient un score d'Apgar $<7$. Cent pour cent des enfants $(n=153)$ avaient été aspirés à la naissance et $24,8 \%(n=38)$ avaient été ventilé au masque. Cent 
vingt-trois malades $(80,3 \%)$ avaient présentés une encéphalopathie anoxoischémique dont 95 (77,2\%) étaient au stade 1; 27 (22,0\%) étaient au stade 2 et $1(0,8 \%)$ était au stade 3 de la classification de Sarnat. Les principaux signes neurologiques étaient les anomalies des réflexes archaïques $(64,2 \%)$; les troubles du tonus $(57,7 \%)$; les troubles de la conscience $(22,8 \%)$; les convulsions (21,9\%). Le taux de létalité était de 10,4\% (16 décès). Les décès étaient associés de façon significative à un accouchement par voie basse, à une RPM, à une ventilation au masque, à une détresse respiratoire et une convulsion. Conclusion: L'AP représente 20,6\% des hospitalisations néonatales à l'hôpital de la paix de Ziguinchor. Sa létalité est de 10,4\%. Elle est associée à une morbidité élevée.

Mots-clés : asphyxie périnatale ; classification de Sarnat ; morbi-mortalité néonatale

\section{Introduction}

L'asphyxie périnatale représente la troisième cause de mortalité néonatale, derrière le faible poids de naissance et les infections néonatales (Thiam, M. (2008).). Elle est définit par une altération sévère des échanges utéroplacentaires conduisant à une hypoxie sévère et une acidose respiratoire puis métabolique. Le diagnostic clinique de l'asphyxie périnatale à la naissance se fait par un score d'APGAR inférieur à 7 à la 5 éme minute ou par l'absence de cri immédiat. La prise en charge de l'asphyxie périnatale est très difficile dans nos pays en développement compte tenu de la faiblesse du plateau technique dans les structures sanitaires et l'insuffisance de formation du personnel médical et paramédical. Au Sénégal elle constitue l'un des principaux motifs de transfert vers les centres hospitaliers de référence comme le service de néonatologie de l'hôpital de la paix de Ziguinchor. L'objectif de ce travail était d'étudier les aspects épidémiologiques, obstétricaux, cliniques et évolutifs de l'asphyxie périnatale à l'hôpital de la paix de Ziguinchor.

\section{Materiels Et Methodes Site de l'étude}

Notre étude a eu pour cadre le service de pédiatrie de l'hôpital de la paix de Ziguinchor. Le service de pédiatrie comporte un pôle d'hospitalisation pour enfants; un centre de réhabilitation et d'éducation nutritionnel (CREN) et un pôle de néonatologie. Ce dernier comporte deux salles : une salle de réanimation néonatale d'une capacité de 8 places dont deux tables chauffantes et de réanimation, une couveuse et 5 berceaux. Une deuxième salle pour une hospitalisation du couple mère enfant. Le personnel 
médical est constitué par un pédiatre assistant universitaire, aidé par deux autres pédiatres praticiens hospitaliers.

\section{Type d'étude}

Il s'agit d'une étude rétrospective, descriptive et analytique portant sur les cas d'asphyxie périnatale hospitalisés dans le service de pédiatrie de l'hôpital de la paix de Ziguinchor, durant la période allant du $1^{\text {er }}$ décembre 2015 au 31 mai 2017.

Population d'étude: elle concerne l'ensemble des nouveau-nés hospitalisés pendant la période d'étude.

\section{Critères d'inclusion}

Etaient inclus tous les nouveau-nés présentant une asphyxie périnatale à la naissance. Le diagnostic d'asphyxie périnatale était fait sur la base d'une anomalie du rythme cardiaque fœtal et/ou d'un liquide amniotique teinté ou méconial ; d'un score d'Apgar $<7$ à la cinquième minute et/ou de l'absence d'un cri immédiat à la naissance ; d'une encéphalopathie anoxo-ischémique.

\section{Critères de non inclusion}

N'étaient pas inclus dans l'étude, les nouveau-nés de mère ayant reçu dans le dernier tiers de la grossesse des neuroleptiques ou des benzodiazépines.

\section{Recueil des données}

Les paramètres étudiés étaient les données épidémiologiques (âge et sexe du nouveau-né, âge de la mère) ; les données obstétricales (nombre de CPN, âge et bilan de la grossesse, complications obstétricales, type de présentation, nature du liquide amniotique, voie d'accouchement). Le comportement immédiat de l'enfant était évalué par le score d'Apgar. Le pronostic néonatal à court terme était évalué sur le plan clinique par la classification de Sarnat. La mortalité était analysée

La saisie et l'analyse des données ont été réalisées avec le logiciel Epi info.

\section{Resultats}

Durant la période d'étude, nous avons colligé 153 cas (90 garçons et 63 filles) d'AP parmi 742 nouveau-nés, soit une incidence de 20,6\% des hospitalisations en néonatologie. L'âge moyen des mères était de 17,2 ans ; $82,0 \%$ des mères avaient un âge compris entre 18 et 35 ans et la moitié d'entre elles $(50,3 \%)$ avaient fait 4 consultations pré natales (CPN) ou plus. 

tableau I.

Les principales complications obstétricales sont représentées dans le

Tableau I : Répartition des nouveau-nés selon les complications obstétricales

\begin{tabular}{|c|c|c|}
\hline Complications obstétricales & Effectif & Pourcentage \\
\hline $\begin{array}{c}\text { HTA gravidique } \\
\mathrm{N}=139 \\
\end{array}$ & 43 & 30,9 \\
\hline $\begin{array}{l}\text { Hématome rétro placentaire } \\
\qquad \mathrm{N}=153\end{array}$ & 07 & 04,6 \\
\hline $\begin{array}{l}\text { Placenta prævia } \\
\mathrm{N}=80\end{array}$ & 01 & 01,3 \\
\hline $\begin{array}{l}\text { Pré éclampsie/Eclampsie } \\
\qquad \mathrm{N}=151\end{array}$ & 15 & 09,9 \\
\hline $\begin{array}{l}\text { Anémie } \\
\mathrm{N}=100\end{array}$ & 37 & 37,0 \\
\hline $\begin{array}{l}\text { Rupture prématurée des membranes } \\
\qquad \mathrm{N}=112\end{array}$ & 32 & 28,6 \\
\hline $\begin{array}{l}\text { Présentation dystocique } \\
\qquad \mathrm{N}=100\end{array}$ & 17 & 17,0 \\
\hline $\begin{array}{l}\text { Anomalie du liquide amniotique } \\
\qquad \mathrm{N}=86\end{array}$ & 06 & 07,0 \\
\hline $\begin{array}{l}\text { Infection maternelle } \\
\qquad \mathrm{N}=11\end{array}$ & 04 & 36,4 \\
\hline $\begin{array}{l}\text { Diabète } \\
\mathrm{N}=114\end{array}$ & 03 & 02,6 \\
\hline $\begin{array}{l}\text { Circulaire du cordon } \\
\mathrm{N}=76\end{array}$ & 02 & 02,6 \\
\hline
\end{tabular}

L'accouchement était fait par césarienne dans $52,3 \%$ des cas et par voie basse dans $47,7 \%$ des cas. Le terme de naissance se situait entre 37 et 41 SA dans $53,3 \%$ des cas. tableau II.

Les principaux aspects obstétricaux et néonataux sont décrits dans le

Tableau II : Répartition des nouveau-nés selon les caractéristiques de l'accouchement

\begin{tabular}{|c|c|c|c|}
\hline Variable & Modalités & Effectif & Pourcentage \\
\hline \multirow{2}{*}{$\begin{array}{c}\text { Nerme de naissance } \\
\text { N=30 }\end{array}$} & $<37$ SA & 12 & 40,0 \\
\cline { 2 - 4 } & $37-41 \mathrm{SA}$ & 16 & 53,3 \\
\cline { 2 - 4 } $\begin{array}{c}\text { Présentation } \\
\mathrm{N}=100\end{array}$ & Céphalique & 02 & 6,7 \\
\cline { 2 - 4 } & Dystocique & 17 & 83,0 \\
\hline $\begin{array}{c}\text { Voie d'accouchement } \\
\mathrm{N}=153\end{array}$ & $\begin{array}{c}\text { Voie basse sans } \\
\text { manœure }\end{array}$ & 65 & 42,0 \\
\cline { 2 - 4 } & $\begin{array}{c}\text { Voie basse avec } \\
\text { manœuvre }\end{array}$ & 08 & 5,2 \\
\cline { 2 - 4 } & Voie haute & 80 & 52,3 \\
\hline Aspect du liquide amniotique & Clair & 35 & 28,7 \\
\hline
\end{tabular}




\begin{tabular}{|c|c|c|c|}
\hline \multirow{2}{*}{$\mathrm{N}=122$} & Méconial & 15 & 12,3 \\
\cline { 2 - 4 } & Teinté & 68 & 55,7 \\
\hline $\begin{array}{c}\text { Score d'Apgar à la 5éme } \\
\text { minute N= 61 }\end{array}$ & $\geq 7$ & 41 & 67,2 \\
\cline { 2 - 4 } & $<7$ & 26 & 42,6 \\
\hline
\end{tabular}

Cent pour cent des nouveau-nés $(n=153)$ avaient été aspirés à la naissance ; $24,8 \%$ d'entre eux $(n=38)$ avaient été ventilés au masque. Cent vingt-trois malades $(80,3 \%)$ avaient présentés une encéphalopathie anoxoischémique dont 95 (77,2\%) étaient au stade 1; 27 (22,0\%) étaient au stade 2 et $1(0,8 \%)$ était au stade 3 de la classification de Sarnat.

Les principaux signes neurologiques étaient les anomalies des réflexes archaïques $(64,2 \%)$, les troubles du tonus $(57,7 \%)$, les troubles de la conscience $(22,8 \%)$, les convulsions $(21,9 \%)$. Les autres complications associées étaient la détresse respiratoire $(31,1 \%)$, l'infection néonatale $(14,3 \%)$, l'état de choc $(1,7 \%)$, les hémorragies $(1,7 \%)$.

La durée moyenne d'hospitalisation était de 10 jours [1 - 32 jours]. Le taux de létalité était de 10,4\% (16 décès). Les décès étaient associés de façon significative à un accouchement par voie basse $(\mathrm{p}=0,010)$, à une RPM de plus de 12 heures $(p=0,041)$, à une ventilation au masque $(p=0,012)$, à une détresse respiratoire $(p=0,030)$, à une convulsion $(p=0,043)$ (tableau III).

Tableau III : Répartition des nouveau-nés selon les facteurs associés au décès

\begin{tabular}{|c|c|c|c|c|c|}
\hline \multirow{2}{*}{ Paramètres } & \multicolumn{2}{|c|}{ Décédé } & \multicolumn{2}{c|}{ Vivant } & $\begin{array}{c}\text { P } \\
\text { value }\end{array}$ \\
\cline { 2 - 6 } & Effectif & Pourcentage & Effectif & Pourcentage & \\
\hline Détresse respiratoire & 07 & 18,4 & 31 & 81,6 & 0,030 \\
\hline Accouchement par voie basse & 08 & 10,9 & 65 & 89,1 & 0,010 \\
\hline Convulsions & 04 & 44,4 & 05 & 55,6 & 0,043 \\
\hline $\begin{array}{c}\text { Ventilation au masque } \\
\text { Rupture prématurée des } \\
\text { membranes }\end{array}$ & 16 & 42,1 & 22 & 57,9 & 0,012 \\
\hline
\end{tabular}

\section{Discussion}

L'AP demeure un problème de santé publique dans les pays en développement. Au Sénégal elle occupe la troisième position, derrière les faibles poids de naissance et les infections dans la mortalité néonatale (Thiam, M. (2008).).

Dans notre étude nous avons noté une incidence élevée à 20,6\%. L'incidence serait de 6,7\% au niveau national et de $13,3 \%$ au niveau de la région de Ziguinchor (EDS-continue 2015). Faye et al. ont retrouvé une 
incidence plus faible que la nôtre à Dakar (8,5\% au centre hospitalier de Pikine et 12\% à l'hôpital Albert Royer) (Faye, P. M. (2015).) (Thiello, A. (2015).). Ailleurs en Afrique, Nimaga et al. au Mali, Klingenberg et al. en Tanzanie, et Bouba et al. au Cameroun rapportent des fréquences faibles (Nimaga, D. (2007).) (Klingenberg, C. (2003).) (Bouba, E. C. (2001).).

L'incidence retrouvée dans notre étude est comparable à celle retrouvée dans d'autres services de néonatologie d'Afrique avec un pôle mère-enfant (Ouédraogo-Yugbaré, S. O. (2015).) (Diarra, K. L. (2008).). Ces différences de fréquence pourraient s'expliquer par la méthodologie du type d'étude (la définition des cas d'AP), la différence des cadres d'étude (pôle mère-enfant).

Dans notre étude, l'AP survenait dans $82,0 \%$ des cas chez les nouveau-nés de mères âgées entre 18 et 35 ans (tranche d'âge dont le taux de fécondité est maximal). Elle survenait également dans 50,3\% des cas chez les nouveau-nés de mères ayant fait au moins $4 \mathrm{CPN}$. Cette population de parturientes est superposable à celle de l'EDS 2015, ou 47\% des gestantes ont réalisé au moins 4 CPN comme le recommande l'OMS pour un bon suivi de la grossesse. Une amélioration du niveau de couverture en CPN, un renforcement des capacités des obstétriciens et des sages-femmes, sont importantes pour la prévention de l'AP à Ziguinchor.

L'un des signes évocateurs de l'AP, est l'aspect méconial ou teinté du LA, retrouvé dans 68\% des cas dans notre étude (LA méconial 12,3\% ; LA teinté 55,7\%). Ces résultats sont comparables à ceux de Ouédraogo et al. au Burkina Faso, avec 71,8\% d'anomalie du LA (Ouédraogo-Yugbaré, S. O. (2015).). Par contre dans d'autres études africaines, le LA teinté ou méconial, est retrouvé dans 100\% des cas (Diarra, K. L. (2008).) (Bordarier, C. (1998).). En effet il existe un lien significatif entre le LA teinté ou méconial avec l'AP (Diarra, K. L. (2008).). Dans ces études, les auteurs ont défini les cas d'AP par un LA teinté ou méconial.

Dans notre série, 42,6\% des malades avaient un score d'Apgar $<7$ à la 5éme minute. Ce résultat est inférieur à celui d'Okoko et al. au Congo Brazzaville $(91,1 \%$ de nouveau-nés avec un score d'Apgar < à 7 à la 5éme minute) (Okoko, K. L. (2016).). Le score d'Apgar est l'élément diagnostique le plus utilisé en Afrique (Diarra, K ; L. (2008).) (Collège National des Gynécologues et Obstétriciens Français. (1997).). Mais selon l'académie américaine de pédiatrie, ce score manque de spécificité (American academy of pediatrics. (2006).). En effet, il est influencé par la prématurité, par l'infection du nouveau-né (Yenan, J. P. (2013).).

La principale complication à court terme de l'AP est l'encéphalopathie anoxo-ischémique. Dans notre étude, elle était présente chez 123 malades, soit 80,4\%. Nos résultats sont plus faibles que ceux d'Okoko et al. au Congo Brazzaville, avec 100\% d'encéphalopathie anoxo- 
ischémique parmi 224 nouveau-nés présentant une AP à la naissance. Les anomalies des réflexes archaïques prédominaient avec 84,9\%, suivies des troubles du tonus $(82,5 \%)$, des troubles de la conscience $(75,3 \%)$ et des convulsions $(26,8 \%)$. Dans cette étude, les nouveau-nés sont reçus en moyenne 2 heures après l'accouchement, délai suffisant pour la survenue de l'encéphalopathie (Okoko, A. R. (2016).).

Les pathologies obstétricales retrouvées dans notre étude, sont conformes à celles retrouvées dans la littérature en Afrique subsaharienne ; la différence résidant parfois dans leur ordre de fréquence (Douba, E. C. (2001).) (Bobossi-Serengbe, G. (2004).) (Bisselele, T. (2013).) (Toure, L. (2012).). Dans notre série, l'anémie maternelle $(37,0 \%)$ occupait la première place, suivie de l'infection maternelle $(36,4 \%)$, de l'HTA $(30,9 \%)$ et de la RPM (28,6\%). L'éclampsie et la pré-éclampsie (9,9\%) occupaient la 5éme place des pathologies maternelles associées à l'AP. Dans l'étude de Diarra, au Mali, l'éclampsie et la pré-éclampsie occupent les deux premières places avec respectivement $21,3 \%$ et $17,2 \%$ (Diarra, K. L. (2008).).

Le taux de létalité de l'AP retrouvé dans notre étude, est moins élevé comparé à ceux d'autres études africaines, 30,4\% au Congo Brazzaville, 31,9\% au Mali (Okoko, A. R. (2016).) (Diarra, K. L. (2008).).

\section{Conclusion}

L'asphyxie périnatale est relativement fréquente à l'hôpital de la paix de Ziguinchor. Elle est associée à une morbi-mortalité élevée. Une meilleure formation des prestataires à la réanimation en salle de naissance et un relèvement du plateau technique du service de réanimation néonatale pourraient améliorer le pronostic.

\section{Conflit d'intérêt}

Les auteurs ne déclarent aucun conflit d'intérêts

\section{Qu'est ce qui est connu sur ce sujet}

L'asphyxie périnatale est responsable d'une mortalité et d'une morbidité néonatale importante dans les pays en développement

\section{Qu'est-ce que votre étude apporte de nouveau}

L'étude précise l'incidence et les facteurs de risques de l'asphyxie périnatale dans un hôpital de référence de province, au sud du Sénégal

\section{References:}

1. Thiam M. La Santé néonatale au Sénégal : à propos d'une enquête communautaire réalisée dans les régions de Thiès, Louga, Kaolack, 
Ziguinchor, et Kolda. [Thèse Méd]. Dakar : Université Cheikh Anta Diop de Dakar ; 2008, №59.

2. Thiello A. Morbidité et mortalité néonatales au centre hospitalier national d'enfants Albert Royer de Dakar. [Thèse Méd].Dakar : Université Cheikh Anta Diop de Dakar ; 2015, N¹47.

3. Faye PM, LY F, Sow A, Ndiaye M, Ba A, Diagne-Gueye NR et al. L'asphyxie périnatale (AP) au service de néonatologie du centre hospitalier de Pikine. 3éme Congrès Nationale de la Société Sénégalaise de Pédiatrie (SOSEPED), 18, 19 et 20 Juin 2015.

4. Nimaga D. Souffrance fœtale aiguë dans la maternité du centre de santé de la commune $\mathrm{V}$ [Thèse Méd]. Bamako: Université de Bamako; 2007, $\mathrm{N}^{\circ} 13$.

5. Klingenberg C, Olomi R, Oneko M, Sam N, Langeland N. Neonatal morbidity and mortality in a Tanzanian tertiary care referral hospital. Ann Trop Paediatr. 2003; 23: 293-9.

6. Douba EC. Souffrance cérébrale asphyxique du nouveau-né à terme au centre mère et enfant de la fondation Chantal Biya de Yaoundé [Thèse Méd]. Yaoundé : Faculté de médecine et des sciences biomédicales de Yaoundé ; 2001, $\mathrm{N}^{\circ} 23$.

7. Bobossi-Serengbe G, Sana-Deyamissi TS, Mbongo-Zindamoyen A, Diemier H, Abanga J, Nadjim-Adim F et al. Morbidité et mortalité néonatales au complexe pédiatrique de Bangui. Med Afr Noire. 2004 ; 5103 : 159-63.

8. Okoko AR, Ekouya-Bowassa G, Moyen E, Togho-Abessou LC, Atanda HL, Moyen G. Asphyxie périnatale au centre hospitalier et universitaire de Brazzaville. J Pediatr Puericul. 2016 ; 29 : 295-300.

9. Ouédraogo-Yugbaré SO, Coulibaly G, Koueta F, Yao S, Savadogo H, Dao $\mathrm{L}$, et al. Profil à risque et pronostic néonatal de l'asphyxie périnatale en milieu hospitalier pédiatrique à Ouagadougou. J Pediatr Puericul. 2015; 28: 64-70.

10. Agence Nationale de la Statistique et de la Démographie (ASND). Enquête Démographique et de Santé Continue au Sénégal EDSContinue 2015. Rapport final 2éme année Dakar, Sénégal.

11. Diarra KL. La souffrance fotale aiguë dans le service de gynécoobstétrique du CHU Gabriel-Touré [Thèse Méd]. Bamako : Université de Bamako; 2008, $\mathrm{N}^{\circ} 43$.

12. Bordarier C, Farhat M. Souffrance cérébrale asphyxique du nouveauné à terme. In: EMC pédiatrie maladies infectieuses. Elsevier Masson SAS; 1998. 1-36 [4-002-S-60].

13. Bisselele T, Naulaers G, Bunga Muntu P, Nkidiaka E, Kapepela M, Mavinga $\mathrm{L}$, et al. A descriptive study of perinatal asphyxia at the 
university hospital of Kinshasa (Democratic Republic of Congo). J Trop Pediatr. 2013; 59: 274-9.

14. American academy of pediatrics, Committee on fetus and newborn, American college of obstetricians and gynecologists and Committee on obstetric practice. The Apgar score. Pediatrics 2006 ; 117 : 14447.

15. Collège National des Gynécologues et Obstétriciens Français Président : Professeur M. Tournaire Extrait des Mises à jour en Gynécologie et Obstétrique - Tome XXI publié le 3.12.1997.

16. Coulibaly G, Ouédraogo-Yugbaré SO, Kouéta F, Yaoc LS, Savadogoc H, Daoa HL et al. Asphyxie périnatale et insuffisance rénale aiguë à Ouagadougou. Arch Pediatr. 2016 ; 23 : 249-54.

17. Yenan JP, Plo KJ, Asse KV. Morbidité et mortalité néonatales dans le service de pédiatrie du CHU de Bouaké. Revue Africaine d'Anesthésiologie et de Médecine d'Urgence. $2013 ; 2: 52$ - 7.

18. Touré L, Wane D, Alford S, Taylor R. Santé maternelle et néonatale au Sénégal, succès et défis. USAID MCHIP (maternal and child health integrated program). Avril 2012. 